01

\title{
Неэмпирический анализ изотопических сдвигов и резонансных эффектов в инфракрасном спектре высокого разрешения фреона-22 ( $\left.\mathrm{CHF}_{2} \mathrm{Cl}\right)$, обогащенного ${ }^{13} \mathrm{C}$
}

\author{
(C) С.В. Краснощеков, ${ }^{1}$ И.К. Гайнуллин, ${ }^{2}$ В.Б. Лаптев, ${ }^{3}$ С.А. Климин, ${ }^{3,}$, \\ ${ }^{1}$ Химический фракультет, МгУ имени М.В. Ломоносова, \\ 119991 Москва, Россия \\ ${ }^{2}$ Физический фракультет, МГУ имени М. В. Ломоносова, \\ 119991 Москва, Россия \\ ${ }^{3}$ Институт спектроскопии РАН, \\ 108840 Москва, Троицк, Россия \\ e-mail: "klimin@isan.troitsk.ru
}

Поступила в редакцию 06.08.2021 г.

В окончательной редакции 01.09.2021 г.

Принята к публикации 15.09.2021 г.

Измерен ИК спектр пропускания изотопной смеси хлордифторметана $\left(\mathrm{CHF}_{2} \mathrm{Cl}\right.$, фреон-22) с $33 \%$ долей изотопа ${ }^{13} \mathrm{C}$ и природным соотношением изотопов хлора в диапазоне частот $1400-740 \mathrm{~cm}^{-1}$ с разрешением $0.001 \mathrm{~cm}^{-1}$ при температуре $20^{\circ} \mathrm{C}$. Расчет структуры и секстичной поверхности потенциальной энергии и поверхностей компонент дипольного момента проведен электронным неэмпирическим квантово-механическим методом Мёллера-Плессета, MP2/cc-pVTZ. Далее потенциал был оптимизирован заменой гармонических частот на частоты, рассчитанные электронным методом связанных кластеров, CCSD(T)/aug-cc-pVQZ. Paсчет фундаментальных и составных частот был проведен с помощью операторной теории возмущений Ван Флека $(\mathrm{CVPT} n)$ второго и четвертого порядка $(n=2,4)$. Резонансные эффекты были смоделированы с помощью дополнительного вариационного расчета в базисе до четырехкратного возбуждения $\mathrm{VCI}(4)$. Среднее отклонение предсказанных фундаментальных частот от экспериментальных для изотопологов ${ }^{12} \mathrm{C}$ составила $\sim 1.5 \mathrm{~cm}^{-1}$. Достигнутая точность позволила достоверно предсказать изотопические сдвиги частот изотопологов ${ }^{13} \mathrm{C}$. Показано, что сильный резонанс Ферми $v_{4} / 2 v_{6}$ доминирует в изотопологах ${ }^{12} \mathrm{C}$ и практически отсутствует в ${ }^{13} \mathrm{C}$. Подтверждено литературное [Spectrochim. Acta A, 44:553] предположение о расщеплении $v_{1}(\mathrm{CH})$ за счет резонанса $v_{1} / v_{2}+v_{7}+v_{9}$. Определены коэффициенты полиадного квантового числа. Проведенный анализ позволил предварительно идентифицировать центры колебательно-вращательных полос изотопологов ${ }^{13} \mathrm{CHF}_{2}{ }^{35} \mathrm{Cl}$ и ${ }^{13} \mathrm{CHF}_{2}{ }^{37} \mathrm{Cl}$ в спектре смеси в рамках подготовки к индивидуальным анализам колебательно-вращательных структур отдельных колебательных переходов.

Ключевые слова: фреон-22, спектроскопия высокого разрешения, ферми-резонанс, операторная теория возмущений, гибридная поверхность потенциальной энергии.

DOI: $10.21883 /$ OS.2022.01.51884.27-21

\section{Введение}

Хлордифторметан $\left(\mathrm{CHF}_{2} \mathrm{Cl}, \mathrm{HCFC}-22\right.$, фреон-22) является широко распространенным химическим веществом, которое в промышленных количествах используется в синтезе фторполимеров, в качестве рабочего вещества в холодильных установках и как вспенивающий агент [1]. Фреон-22 присутствует в атмосфере Земли [2-4] и потенциально может влиять на химические процессы в верхних слоях атмосферы [5]. Поскольку ожидаемое время жизни $\mathrm{CHF}_{2} \mathrm{Cl}$ в тропосфере составляет около 15.6 лет [6], при увеличении темпов выбросов этого соединения [7] можно ожидать возрастания его роли в химии разрушения озона $[5,8]$. Таким образом, важная роль этого соединения в экологии, его возможное влияние на озоновый слой Земли и необходимость мониторинга присутствия этого вещества в атмосфере обусловливает большой интерес к изучению его спектров в ИК диапазоне.

Геометрические параметры молекулы фреона-22 $\left(r_{0^{-}}\right.$, $r_{s}-, r_{z}$-структуры) были получены по микроволновым данным в работе Крэмба с сотрудниками (1988) [9], где также решалась обратная гармоническая колебательная задача. Позднее микроволновые спектры подробно изучались в работах Кисиель с сотрудниками (1995) [10] и Бланко с сотрудниками (1996) [11]. Достоверная равновесная геометрическая структура молекулы была недавно установлена в работе [12] на основе гибридного метода, корректирующего экспериментальные вращательные постоянные с помощью теоретических постоянных колебательно-вращательного взаимодействия.

Молекула фреона-22 обладает симметрией $C_{s}$, все девять ее нормальных мод активны в ИК спектрах поглощения и обладают контурами типа $a / c$ для $v_{1}-v_{6}\left(A^{\prime}\right)$ либо типа $b$ для $v_{7}-v_{9}\left(A^{\prime \prime}\right)$. Впервые ИК спектр низкого 
разрешения фреона-22 в газовой фазе был измерен в работе [13]. Отнесение частот фундаментальных и некоторых составных колебаний и обертонов впервые было выполнено в работе [14], в которой был получен ИК спектр низкого разрешения $\mathrm{CHF}_{2} \mathrm{Cl}$ в жидком аргоне. Далее аналогичные исследования спектров матричной изоляции в $\mathrm{Ar}, \mathrm{Kr}, \mathrm{Xе} \mathrm{проводились} \mathrm{в} \mathrm{работе} \mathrm{[15].} \mathrm{Спектры}$ комбинационного рассеяния света в газовой фазе изучались в работах $[16,17]$, а в жидкой и твердой фазах - в работах [18-22], при этом в работе [16] были измерены абсолютные интенсивности и коэффициенты деполяризации. Спектры среднего разрешения $\left(0.12-0.5 \mathrm{~cm}^{-1}\right)$ в широком диапазоне $\left(15000-350 \mathrm{~cm}^{-1}\right)$, колебательная ангармоничность и резонансы Ферми были подробно изучены в работе Брауна с сотрудниками (1988) [23]. Был зарегистрирован резонанс Ферми $v_{4} / 2 v_{6}$, а также высказано предположение о случайном резонансе $v_{1} / v_{2}+v_{7}+v_{9}[23]$.

Ангармоническое силовое поле фреона-22 было впервые получено в работе Палмиери с сотрудниками (1995) [24], где гармонические силовые постоянные на уровне МР2 комбинировались с кубичными и квартичными, полученными методом Хартри-Фока. В работе Курамшиной с сотрудниками (1994) [25] гармоническое силовое поле фреона-22 было получено комбинированным методом путем решения обратной регуляризованной задачи, стабилизированной неэмпирическим полем на уровне MP2/6-31G*. К настоящему времени расчет поверхности потенциальной энергии нуждается в обновлении в соответствии с современными стандартами (метод MP2 для ангармонической части потенциала и метод связанных кластеров $\operatorname{CCSD}(\mathrm{T})$ для гармонических частот).

В дальнейшем в основном измерялись ИК спектры $\mathrm{CHF}_{2} \mathrm{Cl}$ высокого разрешения (от 0.04 до $0.0007 \mathrm{~cm}^{-1}$ ), которые были получены в газовой фазе или в сверхзвуковых молекулярных пучках для различных фундаментальных колебательно-вращательных полос при разных температурах [26-40]. Колебательно-вращательный анализ полос всех фундаментальных колебательных переходов был завершен к 2002 г. с выходом исследования по $v_{2}$ и $v_{7}[36]$.

Сводка литературных данных по спектроскопии высокого разрешения дана в книге [41]. В существующих работах спектральное разрешение порядка $0.001 \mathrm{~cm}^{-1}$ позволило измерить отдельные линии колебательновращательных переходов, доплеровское уширение которых составляло примерно $0.0015 \mathrm{~cm}^{-1}$ при комнатной температуре [38]. В литературе также проводился теоретический анализ полученных результатов, отнесение линий, исследование резонансов Ферми $\left(v_{4} / 2 v_{6}\right)$ [40] и Кориолиса $\left(v_{3} / v_{8}, v_{6} / v_{9}\right)[38,33]$.

Спектры высокого разрешения изотопологов $\mathrm{CHF}_{2} \mathrm{Cl}$ были получены в основном для молекул с природным содержанием изотопов углерода, водорода и хлора. Природная концентрация изотопа хлора ${ }^{37} \mathrm{C} 1$ достаточно велика $(24.2 \%)$, что позволило при хорошем спектральном разрешении установок $\left(<0.0024 \mathrm{~cm}^{-1}\right)$ и при охлаждении молекул $\mathrm{CHF}_{2} \mathrm{Cl}$ идентифицировать колебательновращательные линии обеих компонент ${ }^{12} \mathrm{CHF}_{2}{ }^{35} \mathrm{C} 1$ и ${ }^{12} \mathrm{CHF}_{2}{ }^{37} \mathrm{C} 1$ [28,32,35-38]. Однако из-за небольшого изотопического сдвига между компонентами с ${ }^{35} \mathrm{C} 1$ и ${ }^{37} \mathrm{C} 1$ (5.01-0.048 $\left.\mathrm{cm}^{-1}[38]\right) \quad P Q R$-структура колебательновращательных полос изотопологов хлора практически полностью перекрывалась, что затрудняло анализ спектра.

Магилл с сотрудниками (1986) [26] получил ИК и КРспектры газа среднего разрешения (до 0.04-0.002 $\mathrm{cm}^{-1}$ ) изотопологов ( ${ }^{13} \mathrm{C}$ - и D-замещенных), выполнил отнесение полос и исследовал резонанс $v_{4} / 2 v_{6}$. Было показано, что изотопические сдвиги спектров изотопологов, замещенных по ${ }^{13} \mathrm{C}$, существенно больше сдвигов по ${ }^{37} \mathrm{Cl}$ и составляют $25.5 \mathrm{~cm}^{-1}$ для $\nu_{3}, 26.5 \mathrm{~cm}^{-1}$ для $v_{8}$ и $20.35 \mathrm{~cm}^{-1}$ для $v_{4}$ [26]. Можно ожидать, что в изотопной смеси, близкой к эквимолярной, интенсивность колебательно-вращательных линий ${ }^{13} \mathrm{CHF}_{2} \mathrm{Cl}$, по крайней мере, для $P$-ветвей полос $v_{3}, v_{8}$ и $v_{4}$ будет сопоставима с интенсивностью линий ${ }^{12} \mathrm{CHF}_{2} \mathrm{Cl}$.

Ранее подробно исследовалась селективная по изотопу ${ }^{13} \mathrm{C}$ ИК многофотонная диссоциация молекул фреона-22 под действием импульсного излучения $\mathrm{CO}_{2}$-лазера [4246]. В конце 90-х-начале 2000-х годов эти работы завершились созданием в России уникальной лазерной технологии и соответствующего производства по обогащению ${ }^{13} \mathrm{C}$, в котором фреон-22 являлся рабочим веществом $[47,48]$. Подробно изучались УФ и ИК фотохимия фреона-22 и эффект внутримолекулярного колебательного перераспределения в этой молекуле под действием лазерных импульсов нано- и фемтосекундной длительности [49-53].

Анализ литературы показывает наличие существенных пробелов в данных, касающихся положений центров колебательных полос ${ }^{13} \mathrm{C}$-замещенного фреона-22, а также более полной и достоверной информации об эффектах ангармоничности: резонансах Ферми, интенсивностях „темных полос“, приобретающих интенсивность за счет перемешивания волновых функций со „светлыми полосами“, изотопических сдвигах. Очевидна необходимость нового исследования таких эффектов, основанная на использовании наиболее точных современных неэмпирических квантово-механических расчетов. Такое исследование представляет собой и самостоятельный интерес, и является необходимым шагом для подготовки прецизионного анализа колебательновращательных спектров ${ }^{13} \mathrm{C}$-замещенного фреона-22, не проводившегося ранее.

Основная цель наших исследований состоит в измерении и детальной расшифровке колебательновращательных ИК спектров высокого разрешения $\left(0.001 \mathrm{~cm}^{-1}\right)$ молекул ${ }^{13} \mathrm{CHF}_{2} \mathrm{Cl}$ в диапазоне частот $1400-740 \mathrm{~cm}^{-1}$, в который попадают полосы фундаментальных колебаний $v_{2}, v_{3}, v_{4}, v_{7}$ и $v_{8}$. Для этого в экспериментах использовалась изотопная смесь ${ }^{12} \mathrm{CHF}_{2} \mathrm{Cl}$ и ${ }^{13} \mathrm{CHF}_{2} \mathrm{Cl}$ с содержанием ${ }^{13} \mathrm{C}$, равным $33 \%$. 


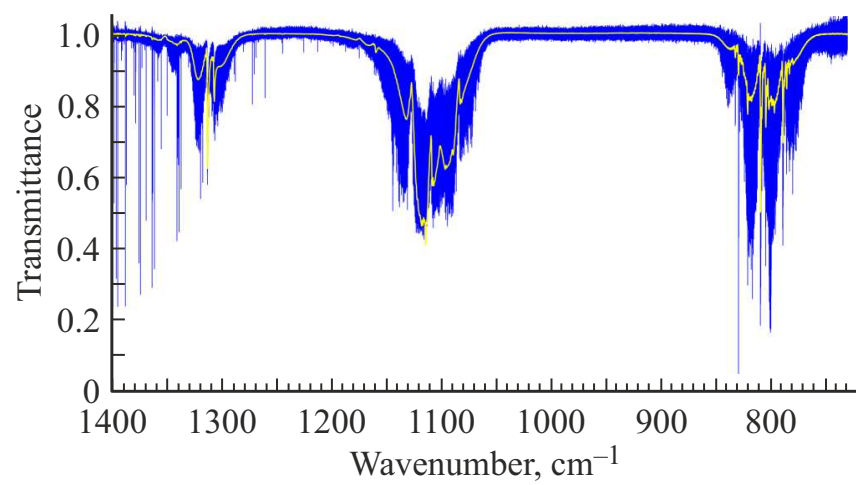

Рис. 1. Инфракрасный спектр высокого разрешения $\left(0.001 \mathrm{~cm}^{-1}\right)$ фреона-22 $\left(\mathrm{CHF}_{2} \mathrm{Cl}\right)$ при давлении 1 Torr и длине кюветы $122 \mathrm{~mm}$ с содержанием ${ }^{13} \mathrm{C}=33 \%$ (синий цвет); светлой линией изображен ИК спектр низкого разрешения $\left(0.1 \mathrm{~cm}^{-1}\right)$ той же изотопной смеси (желтый цвет).

\section{Экспериментальная часть}

Фреон-22, обогащенный по ${ }^{13} \mathrm{C}$ до $33 \%$, был синтезирован А.И. Сорокиным (Пермский филиал АО РНЦ „Прикладная химия“"). Заявленное содержание основного вещества составляло около 99\%, что подтверждалось результатами хроматографического анализа. Контейнер с фреоном-22, обогащенным по ${ }^{13} \mathrm{C}$, подсоединялся к вакуумной системе, которая откачивалась форвакуумным насосом через ловушку с жидким азотом до остаточного давления $7.5 \cdot 10^{-3}$ Torr. К этой же системе подсоединялась газовая кювета длиной $122 \mathrm{~mm}$, объемом $25 \mathrm{~cm}^{3} \mathrm{c}$ окнами из $\mathrm{KBr}$, которые имели вакуумное уплотнение из индия. Все элементы вакуумной системы, включая контейнер и кювету, были изготовлены из нержавеющей стали и тефлона. Кювета заполнялась $\mathrm{CHF}_{2} \mathrm{Cl}$ при давлении 1 Torr и после отсоединения от вакуумной системы фиксировалась в специальном держателе.

Инфракрасные спектры фреона-22, обогащенного по ${ }^{13} \mathrm{C}$ до $33 \%$, были зарегистрированы на фурьеспектрометре BRUKER IFS 125HR с разрешением $0.001 \mathrm{~cm}^{-1}$ с усреднением по 620 сканам при температуре $20^{\circ} \mathrm{C}$. С целью снижения парциального давления углекислого газа и паров воды перед записью спектра объем фурье-спектрометра откачивался вакуумным насосом до остаточного давления порядка 0.05 Торр. Для записи спектров использовались охлаждаемые жидким азотом детекторы на основе $\mathrm{CdHgTe}$ и светоделитель из $\mathrm{KBr}$. При обработке полученные спектры фреона-22 нормировались на спектр пропускания пустой кюветы. Кроме того, шкала частот в диапазоне $1496-829 \mathrm{~cm}^{-1}$ была откорректирована по 12 табулированным линиям воды [54] и двум частотам ${ }^{12} \mathrm{CHF}_{2} \mathrm{Cl}$, измеренным другими авторами в $[28,38]$. После коррекции среднее отклонение указанных 14 частот составило $-3.9 \cdot 10^{-6} \pm 2.5 \cdot 10^{-4} \mathrm{~cm}^{-1}$, что существенно меньше спектрального разрешения.
Таблица 1. Экспериментальные $\left(r_{s}, r_{z}\right)$, полуэмпирические равновесные $\left(r_{e}[12]\right)$ и теоретические геометрические параметры $(\AA, \operatorname{deg})$ молекулы фреона-22

\begin{tabular}{c|c|c|c|c|c|c}
\hline Параметр & $r_{s}^{a}$ & $r_{z}^{a}$ & $\mathrm{MP2}^{\sigma}$ & $\mathrm{CCSD}(\mathrm{T})^{b}$ & $\mathrm{CCSD}(\mathrm{T})^{2}$ & $r_{e}[12]$ \\
\hline$r(\mathrm{C}-\mathrm{H})$ & 1.098 & 1.098 & 1.0841 & 1.0862 & 1.0843 & 1.0850 \\
\hline$r(\mathrm{C}-\mathrm{F})$ & 1.346 & 1.354 & 1.3379 & 1.3384 & 1.3352 & 1.3363 \\
\hline$r(\mathrm{C}-\mathrm{Cl})$ & 1.742 & 1.742 & 1.7627 & 1.7640 & 1.7577 & 1.7560 \\
\hline$\angle \mathrm{HCF})$ & - & - & 109.983 & 110.037 & 109.99 & 109.97 \\
\hline$\angle(\mathrm{HCCl})$ & 110.8 & 110.7 & 109.148 & 109.421 & 109.30 & 109.60 \\
\hline$\angle(\mathrm{FCCl})$ & 110.5 & 111.0 & 109.69 & 109.611 & 109.68 & 109.62 \\
\hline$\angle$ (FCF $)$ & 107.5 & 106.6 & 108.336 & 108.107 & 108.20 & 108.06
\end{tabular}

Примечание. $a-[9] ; \sigma-$ настоящая работа, базисный набор ссpVTZ; в - настоящая работа, базисный набор aug-cc-pVQZ; г метод/базисный набор: $\operatorname{CSD}(\mathrm{T})(\mathrm{ae}) / \mathrm{wCVQZ}$ [12].

На рис. 1 приведен ИК спектр изотопной смеси ${ }^{12} \mathrm{CHF}_{2} \mathrm{Cl}$ и ${ }^{13} \mathrm{CHF}_{2} \mathrm{Cl}$ с содержанием ${ }^{13} \mathrm{CHF}_{2} \mathrm{Cl} 33 \%$ с разрешением $0.001 \mathrm{~cm}^{-1}$. На этом же рисунке на темном фоне спектра высокого разрешения светлой линией изображен ИК спектр низкого разрешения $\left(0.1 \mathrm{~cm}^{-1}\right)$ такой же изотопной смеси. В этом спектре в полосах колебаний $v_{3}$ и $v_{8}$, которые для ${ }^{12} \mathrm{CHF}_{2} \mathrm{Cl}$ сливаются в суммарную полосу с центром на $1116 \mathrm{~cm}^{-1}$ и $v_{4}$ с центром на $809 \mathrm{~cm}^{-1}$, на длинноволновых крыльях можно отчетливо видеть полосы изотопологов ${ }^{13} \mathrm{CHF}_{2} \mathrm{Cl}$.

\section{Методика расчета}

Теоретическая геометрическая структура молекулы фреона-22 была оптимизирована неэмпирическими квантово-механическими методами (базисными наборами) MP2/cc-pVTZ и CCSD(T)/aug-cc-pVQZ с использованием программного комплекса Gaussian'09 [55]. Coпоставление литературных и теоретических геометрических параметров молекулы фреона-22, полученных в данной работе, приведено в табл. 1.

Далее для метода MP2/cc-pVTZ были получены вторые производные энергии по декартовым координатам, которые затем были переведены во внутренние координаты и использованы для расчета нормальных мод всех исследованных изотопологов. Для получения ангармонического секстичного силового поля каждого из изотопологов использовалось численное дифференцирование аналитических вторых производных, рассчитанных на 9точечной сетке для одномерного дифференцирования и 5-точечной сетке для двумерного:

$$
\begin{aligned}
\frac{d}{d x} f(x) & =\frac{1}{840 h}\left[672\left(f_{1}-f_{-1}\right)-168\left(f_{2}-f_{-2}\right)\right. \\
& \left.+32\left(f_{3}-f_{-3}\right)-3\left(f_{4}-f_{-4}\right)\right]
\end{aligned}
$$




$$
\begin{aligned}
& \frac{d^{2} f(x)}{d x^{2}}=-\frac{1}{5040 h^{2}} \\
& \times\left[\begin{array}{c}
14350 f_{0}-8064\left(f_{1}+f_{-1}\right)+1008\left(f_{2}+f_{-2}\right) \\
-128\left(f_{3}+f_{-3}\right)+9\left(f_{4}+f_{-4}\right)
\end{array}\right] \\
& \frac{d^{3} f(x)}{d x^{3}}=\frac{1}{240 h^{3}}\left[488\left(f_{1}-f_{-1}\right)-338\left(f_{2}-f_{-2}\right)\right. \\
& \left.\quad+72\left(f_{3}-f_{-3}\right)-7\left(f_{4}-f_{-4}\right)\right], \\
& \frac{d^{4} f(x)}{d x^{4}}=\frac{1}{240 h^{4}}\left[2730 f_{0}-1952\left(f_{1}+f_{-1}\right)\right. \\
& \left.+676\left(f_{2}-f_{-2}\right)-96\left(f_{3}-f_{-3}\right)+7\left(f_{4}-f_{-4}\right)\right] .
\end{aligned}
$$

Таким образом, максимальное число различных индексов в секстичных силовых постоянных равно четырем. Шаг сетки составил 0.02 в единицах размерных нормальных координат $\left[\AA \cdot u^{1 / 2}\right]$. Полученное силовое поле вкупе с вращательными и кориолисовыми постоянными было использовано для построения колебательного гамильтониана Ватсона.

Колебательное уравнение Шредингера решалось с помощью операторной версии теории возмущений Ван Флека (CVPT $n)$ второго и четвертого порядков $(n=2$, 4) [56-58]. В рамках CVPT исходный гамильтониан в операторной форме, представленный в виде суммы оператора нулевого порядка и возмущений, подвергается последовательности из $K$ унитарных преобразований $U=U_{K} \ldots U_{2} U_{1} H U_{1}^{-1} U_{2}^{-1} \ldots U_{K}^{-1}$, которые приводят его к требуемой форме:

$$
\hat{H}=U H U^{-1}=U_{K} \ldots U_{2} U_{1} H U_{1}^{-1} U_{2}^{-1} \ldots U_{K}^{-1} .
$$

Целью этой последовательности преобразований является коммутируемость преобразованного оператора $\hat{H}$ с оператором $H_{0}$ нулевого порядка, за исключением резонансных членов, которые обрабатываются отдельно в рамках вариационной задачи $\mathrm{VCI}(m)$ в ограниченном базисе волновых функций. Наш опыт показывает, что достаточно использовать базисы с уровнями максимального суммарного возбуждения от двух до четырех колебательных квантов $(m=2,3,4)$.

Унитарные преобразования в методе CVPT определены в экспоненциальной форме, причем эрмитов (самосопряженный) оператор является генератором унитарного преобразования:

$$
U_{k}=\exp \left(i S_{k}\right), \quad U_{k}^{-1} U_{k}=I, \quad S_{k}=-S_{k}^{\dagger} \quad\left\langle S_{k}\right\rangle_{\text {diag }}=0 .
$$

Техника $\operatorname{CVPT}(n)$ подробно описана в литератуpe [56-59]. Например, дважды преобразованный гамильтониан может быть получен после вычисления суммы операторов:

$$
\begin{aligned}
& \hat{H}^{(2)}=H_{0}+H_{1}+i\left[S_{1}, H_{0}\right]+H_{2}+i\left[S_{1}, H_{1}\right] \\
& -\frac{1}{2}\left[S_{1},\left[S_{1}, H_{0}\right]\right]+i\left[S_{2}, H_{0}\right] .
\end{aligned}
$$

Вид генераторов унитарных преобразований отыскивается из однотипных уравнений вида

$$
i\left[S_{K}, H_{0}\right]=\hat{H}_{K}^{(K)}-\hat{H}_{K}^{(K-1)} .
$$

Существенное упрощение формализма CVPT достигается при условии перехода от операторов координаты и импульса к операторам рождения/уничтожения колебательных квантов [57]:

$$
q \rightarrow 2^{-1 / 2}\left(a^{\dagger}+a\right), \quad p \rightarrow 2^{-1 / 2} i\left(a^{\dagger}-a\right) .
$$

В этом случае релевантная часть оператора Гамильтона может быть приведена к нормально упорядоченному виду:

$$
\hat{H}_{K}^{(K-1)}=\sum_{j} h_{j} \prod_{l=1}^{M}\left[\left(a_{l}^{\dagger}\right)^{m_{j l}}\left(a_{l}\right)^{n_{j l}}+\left(a_{l}^{\dagger}\right)^{n_{j l}}\left(a_{l}\right)^{m_{j l}}\right],
$$

а для получения требуемого вида оператора $S_{K}$ необходимо предварительно удалить из этого операторного полинома диагональные $(d)$ и резонансные $(r)$ операторы и применить формулу

$$
\begin{gathered}
S_{k}=-i \sum_{j \neq d, r} h_{i}\left(\sum_{l=1}^{M}\left(m_{j l}-n_{j l}\right) \omega_{l}\right)^{-1} \\
\prod_{l=1}^{M}\left[\left(a_{l}^{\dagger}\right)^{m_{j l}}\left(a_{l}\right)^{n_{j l}}-\left(a_{l}^{\dagger}\right)^{n_{j l}}\left(a_{l}\right)^{m_{j l}}\right] .
\end{gathered}
$$

Коэффициент в круглой скобке перед оператором в уравнении (11) отвечает за условие малости канонического преобразования, обеспечивающего сходимость эффективного гамильтониана данхэмовского типа. Указанный коэффициент безразмерен и в практических вычислениях используется его определенное пороговое значение $\Xi^{\dagger}$ :

$$
\Xi_{k}=\left|h_{k}\left(\sum_{l=1}^{M}\left(m_{k l}-n_{k l}\right) \omega_{l}\right)^{-1}\right|>\Xi^{\dagger},
$$

при превышении которого предполагается возможность колебательного резонанса. Величина частотного знаменателя в (12) отвечает за разность энергий взаимодействующих состояний, которая может быть использована в качестве дополнительного критерия резонанса:

$$
\Delta E=\left|\sum_{l=1}^{M}\left(m_{k l}-n_{k l}\right) \omega_{l}\right| .
$$

Практический расчет ангармонических колебательных состояний и интенсивностей переходов в ИК спектре проводился с использованием программного комплекса ANCO [59-61] на основе полученных поверхностей потенциальной энергии и компонент дипольного момента. Методика расчета была аналогична использованной в 


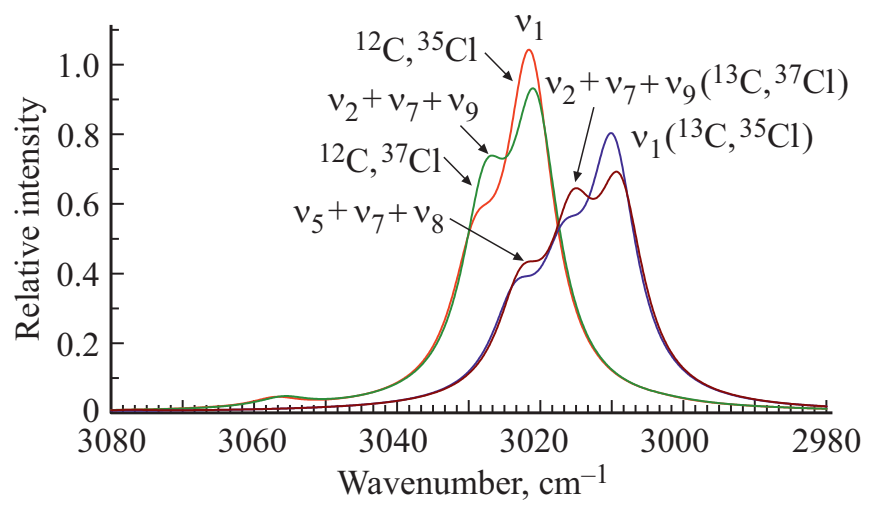

Рис. 2. Суперпозиция расчетных ИК спектров четырех изотопологов $\mathrm{CHF}_{2} \mathrm{Cl}$ в диапазоне $3080-2980 \mathrm{~cm}^{-1}$. Расщепления пиков $v_{1}$ обусловлены случайным резонансом $v_{1} / v_{2}+v_{7}+v_{9}$, а в случае изотопологов ${ }^{13} \mathrm{C}-$ также $v_{1} / \nu_{5}+v_{7}+v_{8}$ (см. текст). Цветовые обозначения: ${ }^{12} \mathrm{C},{ }^{35} \mathrm{Cl}$ - красный, ${ }^{12} \mathrm{C},{ }^{37} \mathrm{Cl}-$ зеленый, ${ }^{13} \mathrm{C},{ }^{35} \mathrm{Cl}-$ синий, ${ }^{13} \mathrm{C},{ }^{37} \mathrm{Cl}$ - коричневый.

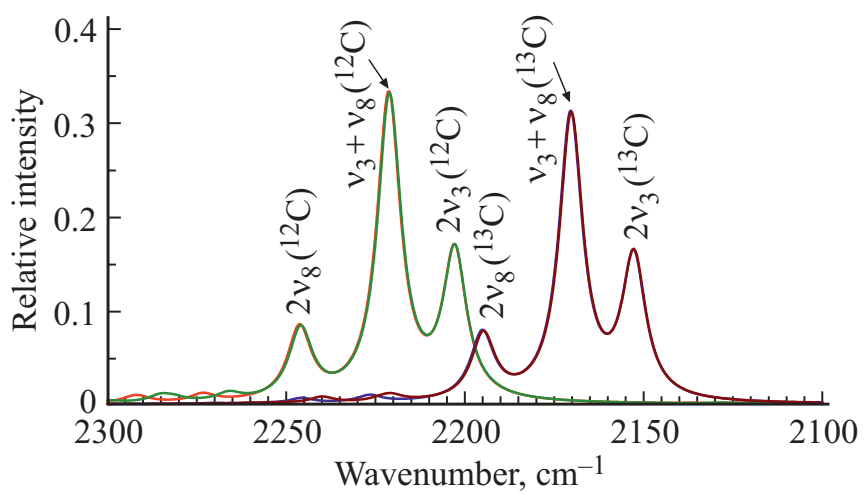

Рис. 3. Суперпозиция расчетных ИК спектров четырех изотопологов $\mathrm{CHF}_{2} \mathrm{Cl}$ в диапазоне $2300-2100 \mathrm{~cm}^{-1}$. Цветовые обозначения: ${ }^{12} \mathrm{C},{ }^{35} \mathrm{Cl}$ - красный, ${ }^{12} \mathrm{C},{ }^{37} \mathrm{Cl}$ - зеленый, ${ }^{13} \mathrm{C},{ }^{35} \mathrm{Cl}-$ синий, ${ }^{13} \mathrm{C},{ }^{37} \mathrm{Cl}$ - коричневый.

предыдущих исследованиях [62-69]. Для графического моделирования итоговых спектров для пика с энергией перехода $E_{j}$ и ИК интенсивностью $I_{j}^{(I R)}(\mathrm{km} / \mathrm{mol})$ использовался контур типа Лоренца,

$$
L_{j}(x)=I_{j}^{I R} w \pi^{-1}\left[\left(E_{j}-x\right)^{2}+w^{2}\right]^{-1},
$$

с фиксированной полушириной $w=4 \mathrm{~cm}^{-1}$. Сканирование всех рассчитанных колебательных уровней с некоторым шагом дискретизации и накоплением суммарной интенсивности в одномерный массив данных образует итоговую форму рассчитанного спектра. Для всех четырех изотопологов наборы предсказанных уровней энергии и их относительные интенсивности представлены в графическом виде на рис. 2-7 (диапазоны 3080-2980, 2300-2100, 1750-1500, 1370-1270, $1200-1000$ и $850-750 \mathrm{~cm}^{-1}$ соответственно).

\section{Результаты и обсуждение}

\section{Теоретические фундаментальные частоты}

Одним из основных теоретических результатов данного исследования является расчет секстичного силового поля четырех изотопологов фреона-22 в нормальных координатах с использованием гибридной техники замены гармонических частот на их аналоги, рассчитанные высокоточным методом связанных кластеров $(\operatorname{CCSD}(\mathrm{T}))$ с использованием достаточно большого базисного набора aug-cc-pVQZ. Расчет фундаментальных частот колебаний двух ${ }^{12} \mathrm{C}$-изотопологов, проведенный с помощью операторной теории возмущений Ван Флека второго порядка и дополненный вариационной задачей в четырехкратно возбужденном базисе $\mathrm{VCI}(4)$ для учета резонансных эффектов, показывает, что теоретические значения частот совпадают с экспериментальными положениями центров полос со среднеквадратичной ошибкой порядка $1.5 \mathrm{~cm}^{-1}$ (табл. 2 и 3). Примечательно, что

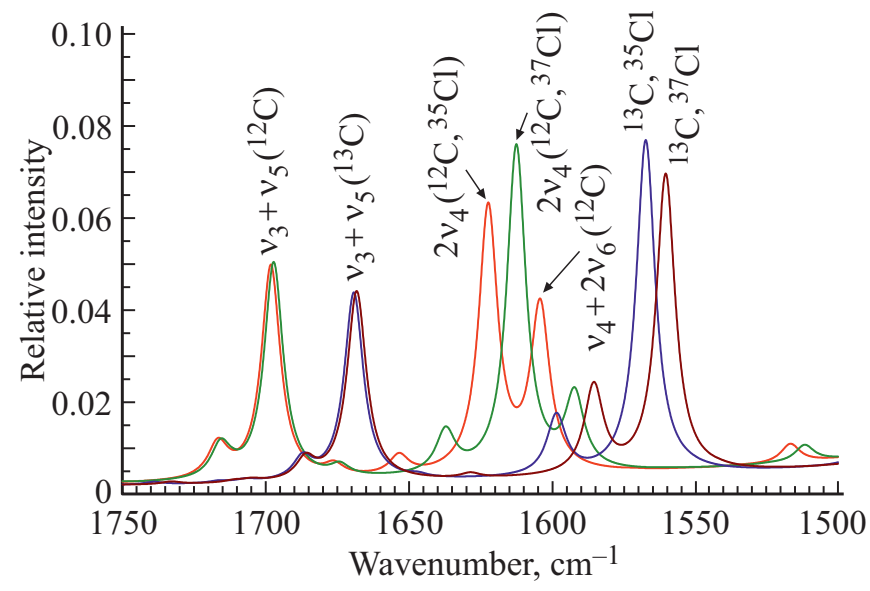

Рис. 4. Суперпозиция расчетных ИК спектров четырех изотопологов $\mathrm{CHF}_{2} \mathrm{Cl}$ в диапазоне $1750-1500 \mathrm{~cm}^{-1}$. Цветовые обозначения: ${ }^{12} \mathrm{C},{ }^{35} \mathrm{Cl}$ - красный, ${ }^{12} \mathrm{C},{ }^{37} \mathrm{Cl}$ - зеленый, ${ }^{13} \mathrm{C},{ }^{35} \mathrm{Cl}-$ синий, ${ }^{13} \mathrm{C},{ }^{37} \mathrm{Cl}$ - коричневый.

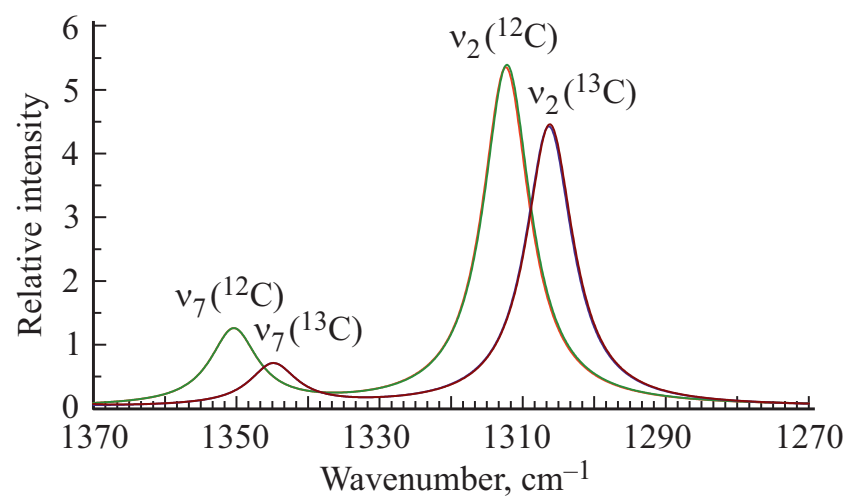

Рис. 5. Суперпозиция расчетных ИК спектров четырех изотопологов $\mathrm{CHF}_{2} \mathrm{Cl}$ в диапазоне $1370-1270 \mathrm{~cm}^{-1}$. Цветовые обозначения: ${ }^{12} \mathrm{C},{ }^{35} \mathrm{Cl}$ - красный, ${ }^{12} \mathrm{C},{ }^{37} \mathrm{Cl}-$ зеленый, ${ }^{13} \mathrm{C},{ }^{35} \mathrm{Cl}-$ синий, ${ }^{13} \mathrm{C},{ }^{37} \mathrm{Cl}$ - коричневый. 


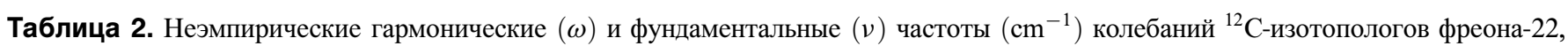
рассчитанные с помощью методов CVPT2/VCI(4) (PT2) и CVPT4/VCI(4) (PT4) с использованием ангармонического гибридного потенциала MP2/cc-pVTZ и CCSD(T)/aug-cc-pVQZ (см. текст)

\begin{tabular}{|c|c|c|c|c|c|c|c|}
\hline$v_{k}$ & Отнесение & $\omega, \mathrm{MP} 2$ & $\omega, \mathrm{CC}$ & $v$, PT2 & RMSE & v, эксперимент & $v$, PT4 \\
\hline \multicolumn{8}{|c|}{$A^{\prime}{ }^{12} \mathrm{CHF}_{2}{ }^{35} \mathrm{Cl}$} \\
\hline$v_{1}$ & $v(\mathrm{CH})$ симметричное валентное & 3193.50 & 3156.16 & 3021.45 & -0.19 & $3021.6335[42]$ & 3021.64 \\
\hline$v_{2}$ & $\delta(\mathrm{ClCH})$ деформационное & 1354.65 & 1340.89 & 1312.35 & -0.74 & $1313.0936[36]$ & 1311.91 \\
\hline$v_{3}$ & $v\left(\mathrm{CF}_{2}\right)$ симметричное валентное & 1142.21 & 1130.91 & 1107.46 & -1.27 & $1108.7293[39]$ & 1107.70 \\
\hline$v_{4}$ & $v(\mathrm{CCl})$ валентное & 831.47 & 822.94 & 806.52 & -2.75 & $809.2726[28]$ & 806.57 \\
\hline$v_{5}$ & $\chi\left(\mathrm{CF}_{2}\right)$ ножничное & 607.04 & 601.65 & 595.02 & -1.35 & $596.3714[31]$ & 594.99 \\
\hline$v_{6}$ & $\delta(\mathrm{FCCl})$ деформационное & 420.63 & 415.95 & 410.64 & -2.29 & $412.9285[34]$ & 410.68 \\
\hline \multicolumn{8}{|c|}{$A^{\prime \prime}{ }^{12} \mathrm{CHF}_{2}{ }^{35} \mathrm{Cl}$} \\
\hline$v_{7}$ & $\rho\left(\mathrm{CF}_{2}\right)$ маятниковое & 1402.35 & 1384.19 & 1350.39 & -1.31 & $1351.7019[36]$ & 1350.23 \\
\hline$v_{8}$ & $v\left(\mathrm{CF}_{2}\right)$ асимметричное валентное & 1173.42 & 1157.07 & 1127.09 & -0.20 & $1127.2851[39]$ & 1127.87 \\
\hline$v_{9}$ & $\chi\left(\mathrm{CF}_{2}\right)$ крутильное & 372.85 & 367.91 & 364.26 & -1.94 & $366.1972[34]$ & 364.25 \\
\hline \multicolumn{8}{|c|}{$A^{\prime}{ }^{12} \mathrm{CHF}_{2}{ }^{37} \mathrm{Cl}$} \\
\hline$v_{1}$ & $v(\mathrm{CH})$ симметричное валентное & 3193.50 & 3156.16 & 3020.72 & -0.68 & $3021.3995[42]$ & 3020.73 \\
\hline$v_{2}$ & $\delta(\mathrm{ClCH})$ деформационное & 1354.56 & 1340.81 & 1312.18 & -0.71 & $1312.8941[36]$ & 1311.66 \\
\hline$v_{3}$ & $v\left(\mathrm{CF}_{2}\right)$ симметричное валентное & -1.27 & $1108.6813[39]$ & 1107.66 & & & \\
\hline$v_{4}$ & $v(\mathrm{CCl})$ валентное & 828.42 & 819.88 & 801.35 & -3.15 & $804.502[28]$ & 801.40 \\
\hline$v_{5}$ & $\chi\left(\mathrm{CF}_{2}\right)$ ножничное & 606.14 & 600.79 & 594.15 & -1.25 & $595.4[30]$ & 594.12 \\
\hline$v_{6}$ & $\delta(\mathrm{FCCl})$ деформационное & 415.51 & 410.88 & 405.65 & -2.27 & $407.9182[34]$ & 405.69 \\
\hline \multicolumn{8}{|c|}{$A^{\prime \prime}{ }^{12} \mathrm{CHF}_{2}{ }^{37} \mathrm{Cl}$} \\
\hline$v_{7}$ & $\rho\left(\mathrm{CF}_{2}\right)$ маятниковое & 1402.34 & 1384.18 & 1350.37 & -1.31 & $1351.6814[36]$ & 1350.16 \\
\hline$v_{8}$ & $v\left(\mathrm{CF}_{2}\right)$ асимметричное валентное & 1173.42 & 1157.06 & 1126.85 & -0.28 & $1127.1227[39]$ & 1127.62 \\
\hline$v_{9}$ & $\chi\left(\mathrm{CF}_{2}\right)$ крутильное & 370.87 & 365.96 & 362.35 & -1.92 & $364.2667[34]$ & 362.35 \\
\hline
\end{tabular}

все ошибки имеют систематический красный сдвиг, что однозначно свидетельствует о наличии неучтенных вкладов в рассчитанное силовое поле. Полученный результат без сомнения является успехом в неэмпирических расчетах колебательных спектров многоатомных молекул, который было трудно прогнозировать в конце 20-го века при отсутствии реализаций достаточно продвинутых и экономически целесообразных квантово-механических методов расчета силового поля. Фактическая ошибка предсказания фундаментальных частот лишь немного уступает пороговой величине „спектроскопической точности“ в размере одного обратного сантиметра [70], и этот результат достигнут для пятиатомных молекул, содержащих 48 электронов.
Полученное секстичное силовое поле и численноаналитическая реализация операторной теории возмущений Ван Флека позволили рассчитать значения фундаментальных частот в четвертом порядке. Их анализ показывает, что за исключением $v_{1}$ для изотополога ${ }^{13} \mathrm{C},{ }^{37} \mathrm{Cl}$, сдвиги частот по сравнению со вторым порядком не превышают $0.8 \mathrm{~cm}^{-1}$, а среднеквадратичная ошибка улучшается весьма незначительно. Это дает основание считать результаты, полученные во втором порядке теории возмущений, достаточно достоверными и взять их за основу теоретической модели для дальнейшего сравнения теории с экспериментом и проведения предсказаний. Однако в упомянутом случае $v_{1}$ для $\left({ }^{13} \mathrm{C},{ }^{37} \mathrm{Cl}\right)$ наблюдается синий сдвиг на $5.8 \mathrm{~cm}^{-1}$, что объясняется перераспределением вкладов волно- 
Таблица 3. Неэмпирические гармонические $(\omega)$ и фундаментальные $(v)$ частоты $\left(\mathrm{cm}^{-1}\right)$ колебаний ${ }^{13} \mathrm{C}$-изотопологов фреона-22, рассчитанные с помощью методов CVPT2/VCI(4) (PT2) и CVPT4/VCI(4) (PT4) с использованием ангармонического гибридного потенциала MP2/cc-pVTZ и CCSD(T)/aug-cc-pVQZ (см. текст); экспериментальные данные взяты из работы [26]

\begin{tabular}{|c|c|c|c|c|c|c|c|}
\hline$v_{k}$ & Отнесение & $\omega, \mathrm{MP} 2$ & $\omega, \mathrm{CC}$ & $v, \mathrm{PT} 2$ & RMSE & v, эксперимент & $v$, PT4 \\
\hline \multicolumn{8}{|c|}{$A^{\prime}{ }^{12} \mathrm{CHF}_{2}{ }^{35} \mathrm{Cl}$} \\
\hline$v_{1}$ & $v(\mathrm{CH})$ симметричное валентное & 3183.46 & 3146.29 & 3009.87 & -0.73 & 3010.6 & 3009.94 \\
\hline$v_{2}$ & $\delta(\mathrm{ClCH})$ деформационное & 1348.86 & 1335.00 & 1306.33 & -0.87 & 1307.2 & 1305.90 \\
\hline$v_{3}$ & $v\left(\mathrm{CF}_{2}\right)$ симметричное валентное & 1115.72 & 1104.64 & 1082.17 & -1.33 & 1083.5 & 1083.45 \\
\hline$v_{4}$ & $v(\mathrm{CCl})$ валентное & 808.12 & 799.97 & 786.65 & -2.25 & 788.9 & 786.66 \\
\hline$v_{5}$ & $\chi\left(\mathrm{CF}_{2}\right)$ ножничное & 602.72 & 597.38 & 591.07 & -1.23 & 592.3 & 591.04 \\
\hline$v_{6}$ & $\delta(\mathrm{FCCl})$ деформационное & 420.39 & 415.69 & 410.48 & -2.32 & 412.8 & 410.51 \\
\hline \multicolumn{8}{|c|}{$A^{\prime \prime}{ }^{13} \mathrm{CHF}_{2}{ }^{35} \mathrm{Cl}$} \\
\hline$v_{7}$ & $\rho\left(\mathrm{CF}_{2}\right)$ маятниковое & 1395.95 & 1378.18 & 1344.85 & -1.15 & 1346 & 1344.65 \\
\hline$v_{8}$ & $v\left(\mathrm{CF}_{2}\right)$ асимметричное валентное & 1146.06 & 1129.90 & 1101.32 & 0.32 & 1101 & 1102.05 \\
\hline$v_{9}$ & $\chi\left(\mathrm{CF}_{2}\right)$ крутильное & 371.35 & 366.42 & 362.87 & -0.13 & 363 & 362.86 \\
\hline \multicolumn{8}{|c|}{$A^{\prime}{ }^{13} \mathrm{CHF}_{2}{ }^{37} \mathrm{Cl}$} \\
\hline$v_{1}$ & $v(\mathrm{CH})$ симметричное валентное & 3183.46 & 3146.2 & 3008.87 & -1.73 & 3010.6 & 3014.70 \\
\hline$v_{2}$ & $\delta(\mathrm{ClCH})$ деформационное & 1348.76 & 1334.90 & 1306.19 & -1.01 & 1307.2 & 1305.68 \\
\hline$v_{3}$ & $v\left(\mathrm{CF}_{2}\right)$ симметричное валентное & 1115.72 & 1104.63 & 1082.11 & -1.39 & 1083.5 & 1082.41 \\
\hline$v_{4}$ & $v(\mathrm{CCl})$ вал. & 804.98 & 796.80 & 783.22 & -2.28 & 785.5 & 783.24 \\
\hline$v_{5}$ & $\chi\left(\mathrm{CF}_{2}\right)$ ножничное & 601.85 & 596.55 & 590.22 & -1.28 & 591.5 & 590.18 \\
\hline$v_{6}$ & $\delta(\mathrm{FCCl})$ деформационное & 415.27 & 410.63 & 405.50 & -2.30 & 407.8 & 405.53 \\
\hline \multicolumn{8}{|c|}{$A^{\prime \prime}{ }^{13} \mathrm{CHF}_{2}{ }^{37} \mathrm{Cl}$} \\
\hline$v_{7}$ & $\rho\left(\mathrm{CF}_{2}\right)$ маятниковое & 1395.94 & 1378.17 & 1344.84 & -1.16 & 1346 & 1344.59 \\
\hline$v_{8}$ & $v\left(\mathrm{CF}_{2}\right)$ асимметричное валентное & 1146.05 & 1129.90 & 1101.12 & 0.12 & 1101 & 1101.89 \\
\hline$v_{9}$ & $\chi\left(\mathrm{CF}_{2}\right)$ крутильное & 369.36 & 364.46 & 360.95 & -2.05 & 363 & 360.93 \\
\hline
\end{tabular}

вых функций нулевого приближения при резонансе $v_{1} / v_{2}+v_{7}+v_{9}$.

\section{Колебательные резонансы и полиадное квантовое число}

Как показывает расчет в рамках нашей теоретической модели, ряд фундаментальных частот основной молекулы $\left({ }^{12} \mathrm{C},{ }^{35} \mathrm{Cl}\right)$ подвержены резонансным эффектам различной силы. В табл. 4 дана сводка теоретически предсказанных колебательных резонансов основной молекулы фреона-22 $\left({ }^{12} \mathrm{C},{ }^{35} \mathrm{Cl}\right)$, упорядоченных по убыванию параметра „силы резонанса“ (12).

Если рассматривать степени операторов для колебательных мод в качестве целочисленных векторных компонент, то полученный набор векторов может быть использован для построения вектора коэффициентов полиадного квантового числа, ортогонального резонансным векторам [61,71] (в нашем случае оператор $3^{+2} 8^{-2}$ является линейно зависимым):

$$
\begin{aligned}
P= & 8 v_{1}+3 v_{2}+3 v_{3}+2 v_{4}+2 v_{5} \\
& +v_{6}+3 v_{7}+3 v_{8}+v_{9} .
\end{aligned}
$$

Аналогичные наборы полиадных коэффициентов могут быть получены и для других изотопологов помимо основной молекулы. Предварительный анализ показывает, что небольшая вариация в силе резонансов оказывает влияние на конечный вид таких полиадных коэффициентов.

Из табл. 4 видно, что $v_{2}, v_{4}, v_{8}$ основной молекулы участвуют в резонансах Ферми, а обертоны $2 v_{2}, 2 v_{3}, 2 v_{7}, 2 v_{8}$ участвуют в резонансах Дарлинга-Деннисона. Рассмотрим теперь более 


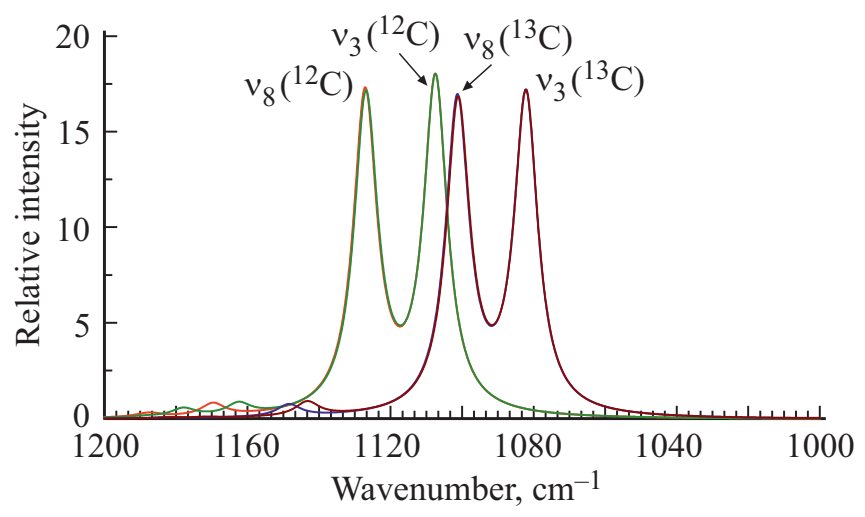

Рис. 6. Суперпозиция расчетных ИК спектров четырех изотопологов $\mathrm{CHF}_{2} \mathrm{Cl}$ в диапазоне $1200-1000 \mathrm{~cm}^{-1}$. Цветовые обозначения: ${ }^{12} \mathrm{C},{ }^{35} \mathrm{Cl}$ - красный, ${ }^{12} \mathrm{C},{ }^{37} \mathrm{Cl}$ - зеленый, ${ }^{13} \mathrm{C},{ }^{35} \mathrm{Cl}-$ синий, ${ }^{13} \mathrm{C},{ }^{37} \mathrm{Cl}$ - коричневый.

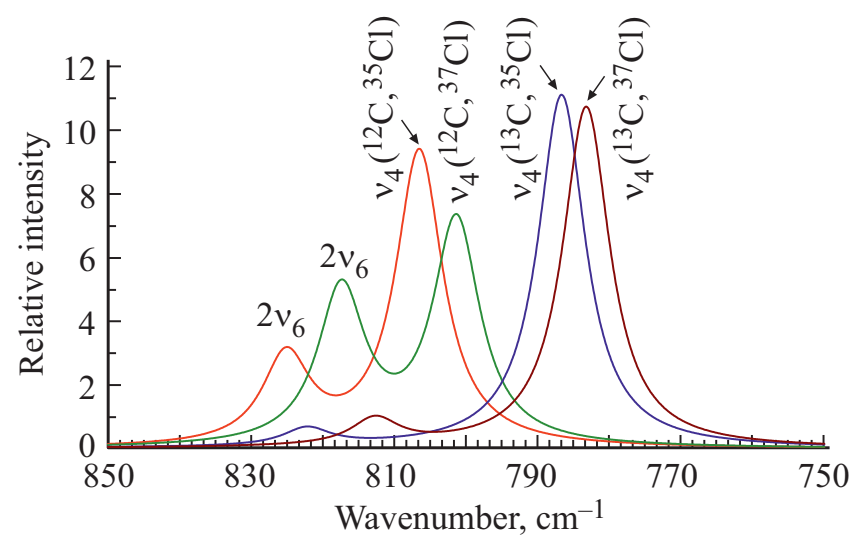

Рис. 7. Суперпозиция расчетных ИК спектров четырех изотопологов $\mathrm{CHF}_{2} \mathrm{Cl}$ в диапазоне $850-750 \mathrm{~cm}^{-1}$. Цветовые обозначения: ${ }^{12} \mathrm{C},{ }^{35} \mathrm{Cl}$ - красный, ${ }^{12} \mathrm{C},{ }^{37} \mathrm{Cl}$ - зеленый, ${ }^{13} \mathrm{C},{ }^{35} \mathrm{Cl}-$ синий, ${ }^{13} \mathrm{C},{ }^{37} \mathrm{Cl}$ - коричневый.

подробно три резонанса, в которых участвуют фундаментальные частоты $v_{1}, v_{2}, v_{4}, v_{8}$. С точки зрения теории большой интерес представляет интерпретация структуры дублета 3024.55(?)-3021.27(v $\left.v_{1}\right)$ с узким расщеплением $\sim 3 \mathrm{~cm}^{-1}$, который обсуждался в ряде работ [23,27,32,72]. Маккензи (1984) [72] идентифицировал эти пики как $Q$-ветви, неявно предполагая резонанс. В хронологически второй работе [23] выдвигались два предположения о природе этого дублета взаимодействие с „горячим“ переходом $\left(v_{1}+v_{9}\right)-v_{9}$ либо с трехквантовым состоянием $v_{2}+v_{7}+v_{9}$. Весьма подробное обсуждение природы указанного дублета приведено в последующей работе Амрейна с сотрудниками (1988) [27], в которой процитированы обе предыдущие, а из пяти возможных объяснений наиболее вероятными признаны (а) взаимодействие $v_{1}$ с ,горячим“ переходом, (б) кориолисов резонанс при высоких значениях $J$. Наконец, Фразер с сотрудниками (1992) [32] в результате детального анализа колебательно-вращательного спектра высокого разрешения констатировал, что вопрос
Таблица 4. Теоретические характеристики ${ }^{*}$ колебательных резонансов фреона- $22\left({ }^{12} \mathrm{C},{ }^{35} \mathrm{Cl}\right)$

\begin{tabular}{c|c|c|c|c|c}
\hline № & $\bar{\omega}_{j}$ & $\Delta_{j}$ & $h_{j}$ & $\Xi_{j}$ & $\hat{R}_{j}$ \\
\hline 1 & 830.7 & 11.4 & 5.57 & 0.4906 & $4^{+1} 6^{-2}$ \\
2 & 3150.4 & 9.9 & 4.43 & 0.4469 & $1^{-1} 5^{+1} 7^{+1} 8^{+1}$ \\
3 & 1175.9 & 37.8 & 8.68 & 0.2297 & $4^{+1} 8^{-1} 9^{+1}$ \\
4 & 2289.1 & 49.9 & 6.31 & 0.1265 & $3^{+2} 8^{-2}$ \\
5 & 2508.0 & 19.0 & 2.35 & 0.1238 & $2^{+1} 3^{-1} 7^{-1} 8^{+1}$ \\
6 & 2727.0 & 87.9 & 10.73 & 0.1221 & $2^{-2} 7^{+2}$ \\
7 & 1292.3 & 98.4 & 10.86 & 0.1104 & $2^{-1} 4^{+1} 6^{+1}$ \\
8 & 1985.2 & 6.4 & 0.61 & 0.0947 & $4^{+1} 5^{-1} 7^{-1} 8^{+1}$ \\
9 & 1538.5 & 23.4 & 2.13 & 0.0911 & $3^{+1} 6^{+1} 8^{-1} 9^{-1}$
\end{tabular}

* Примечание. $\bar{\omega}_{j}-$ средняя гармоническая частота состояний, участвующих в резонансе; $\Delta_{j}$ - гармонический знаменатель в выражении для S-оператора (см. уравнения $(11)-(13)) ; h_{j}-$ константа перед (преобразованным) резонансным оператором; $\Xi$ - „сила резонанса“, частное $h_{j} \cdot \Delta_{j}^{-1} ; \hat{R}_{j}$ - резонансный оператор (без множителя $h_{j}$ ).

о природе указанного расщепления остается открытым и требует дополнительного экспериментального анализа при более высоких температурах.

Неэмпирический колебательный расчет, проведенный нами, позволяет прояснить вопрос о природе дублета $Q$ ветвей 3024.55-3021.27, наблюдаемого в основной молекуле и являющегося результатом резонанса $v_{1} \mathrm{c}$, ,темным“ переходом. Действительно, значение ангармонической силовой постоянной $\varphi_{1279}=4.2 \mathrm{~cm}^{-1}$ допускает появление чисто колебательного резонанса $v_{1} / v_{2}+v_{7}+v_{9}$, возможность которого была озвучена Брауном с сотрудниками (1988) [23] и отвергнутым Амрейном с сотрудниками (1988) [27]. Наш расчет CVPT2/VCI(4) подтверждает наличие этого колебательного резонанса, хотя расчетное расщепление $\left(7.7 \mathrm{~cm}^{-1}\right)$ несколько превышает экспериментальную величину. При этом предсказанное значение $v_{1}=3021.4 \mathrm{~cm}^{-1}$ находится близко к наблюдаемому. Аналогичный резонанс также предсказан для изотополога ${ }^{13} \mathrm{C},{ }^{37} \mathrm{Cl}$. Расчет предсказывает еще один интересный эффект - образование резонансного триплета $v_{1} / v_{2}+v_{7}+v_{9} / v_{5}+v_{7}+v_{8}$ в случае обоих изотопологов ${ }^{13} \mathrm{C}$, что можно видеть на рис. 2. Это предсказание должно быть сопоставлено с экспериментальными данными, что может быть предметом дальнейших исследований.

Столь же важным является резонанс Ферми $v_{4} / 2 v_{6}$, который имеет наибольшее значение параметра „силы резонанса“ $\Xi=0.4906[61,73]$. Этот резонанс был подробно изучен в ряде работ $[14,26,23,28,24,40]$. При этом, однако, отсутствовали данные о поведении этого резонанса в изотопологах ${ }^{13} \mathrm{C}$. Теоретическое значение силовой постоянной $\varphi_{466}=61.5 \mathrm{~cm}^{-1}$ отлично согласуется с экспериментально найденной постоянной резонанса Ферми $W=\varphi_{466} / 4=15.5 \mathrm{~cm}^{-1}$, найденной в работе [23]. В этом исследовании [23] идентифицировано большое число горячих переходов, ассоциированных с 


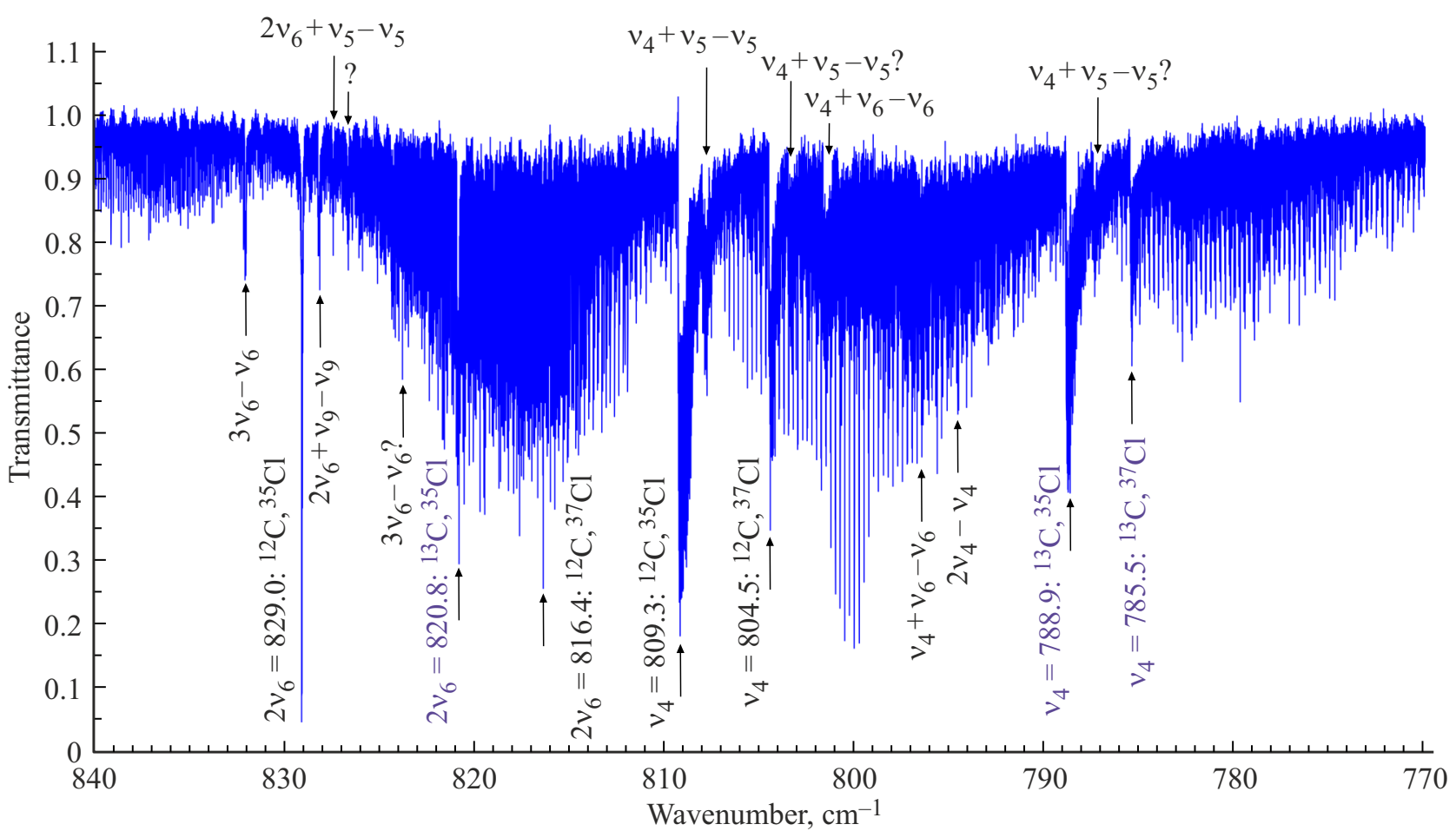

Рис. 8. Фрагмент ИК спектра фреона- 22 высокого разрешения, обогащенного $(33 \%)$ изотопологами ${ }^{13}$ С. На рисунке фиолетовым цветом указаны два пика $v_{4}$ и пик $2 v_{6}$, относящиеся к изотопологам ${ }^{13}$ С. Отнесение ряда „горячих“ переходов указано согласно работе Брауна с сотрудниками (1988) [23].

партнерами резонанса Ферми $v_{4} / 2 v_{6}$. В полученном нами спектре высокого разрешения (рис. 8) также отчетливо видны ранее наблюденные [23] пики $832.0\left(3 v_{6}-v_{6}\right)$, $828.1\left(2 v_{6}+v_{9}-v_{9}\right)$ и $827.4 \mathrm{~cm}^{-1}\left(2 v_{6}+v_{5}-v_{5}\right)$ изотополога ${ }^{12} \mathrm{C},{ }^{35} \mathrm{Cl}$, а также пик $824.2 \mathrm{~cm}^{-1}\left(3 v_{6}-v_{6}\right)$ изотополога ${ }^{12} \mathrm{C},{ }^{37} \mathrm{Cl}$. В этом диапазоне могут находиться аналогичные пики, соответствующие горячим переходам изотопологов ${ }^{13} \mathrm{C}$, но их подробное изучение является предметом отдельной работы. Касательно резонанса Ферми для изотопологов ${ }^{13} \mathrm{C}$ расчет показывает его существенное ослабление при замещении углерода на более тяжелый, что видно на симулированном спектре на рис. 7. Сопоставление расчета $\left(822.2 \mathrm{~cm}^{-1}\right)$ с экспериментальным спектром дает возможность отнести пик $820.8 \mathrm{~cm}^{-1}$ к резонансному обертону $2 v_{6}$ изотополога ${ }^{13} \mathrm{C},{ }^{35} \mathrm{Cl}$.

Как видно из табл. 4, теоретический расчет предсказывает наличие слабого резонанса Ферми $v_{2} / v_{4}+v_{6}$, которому отвечает силовая постоянная $\varphi_{246}=30.8 \mathrm{~cm}^{-1}$. В работе [36] высказано предположение, что экспериментальные свидетельства о резонансных факторах, влияющих на $v_{2}$, могут объясняться влиянием $v_{5}+v_{9}$. Однако наш анализ скорее объясняет указанные экспериментальные факты образованием резонансной триады $v_{2} / v_{4}+v_{6} / 3 v_{6}$, в которой участвует возбужденный резонанс $v_{4} / 2 v_{6}$.

Хотя теоретическая модель предсказывает наличие резонанса $v_{8} / v_{4}+v_{9}$ (табл. 4), его эксперимен- тальное изучение затруднено наличием колебательновращательного кориолисова резонанса $v_{3} / v_{8}$, который подробно изучался в работах $[27,29,35,38,39]$. Согласно нашему расчету, в основной молекуле ${ }^{12} \mathrm{C},{ }^{35} \mathrm{Cl}$ образуется триада $v_{8} / v_{4}+v_{9} / 2 v_{6}+v_{9}$ $\left(1127.1 / 1169.6 / 1187.1 \mathrm{~cm}^{-1}\right)$, при этом составной тон $v_{4}+v_{9}$ увеличивает интенсивность от 0.12 до $8.2 \mathrm{~km} / \mathrm{mol}$, а состояние $2 v_{6}+v_{9}$ - от 0.06 до $2.4 \mathrm{~km} / \mathrm{mol}$. Наш спектр высокого разрешения показывает наличие трех $Q$-ветвей в этом диапазоне с примерными значениями волновых чисел $1159.3,1160.7,1174.6$ и $1187.0 \mathrm{~cm}^{-1}$, которые могут быть интерпретированы как экспериментальное проявление соответствующих резонансных состояний смеси изотопологов, в то время как в спектре низкого разрешения только пик $1159.3 \mathrm{~cm}^{-1}$ является достаточно острым. В работе [26] в спектре низкого разрешения отмечалось наличие близких по частоте пиков $1178 \mathrm{~cm}^{-1}$ для изотопологов ${ }^{12} \mathrm{C}$ и $1181.1 \mathrm{~cm}^{-1}$ для ${ }^{13} \mathrm{C}$. Нам не удалось найти данные по колебательным переходам в этой области спектра среди колебательновращательных исследований высокого разрешения. Данный факт доказывает важность предварительной теоретической информации о колебательных эффектах, которая может быть использована в последующих исследованиях колебательно-вращательной структуры в этой области спектра. 


\section{Многоквантовые переходы}

Как видно из рис. 3, в диапазоне $2300-2100 \mathrm{~cm}^{-1}$ наблюдаются по три составных перехода для каждого изотополога, $2 v_{8}, v_{3}+v_{8}\left(A^{\prime \prime}\right), 2 v_{3}$. Все эти переходы имеют интенсивность, достаточную для экспериментального обнаружения без заимствования от „светлых“ переходов. Например, для основного изотополога интенсивность равна $4.18 \mathrm{~km} / \mathrm{mol}$. Эти переходы наблюдались экспериментально (см. спектрограмму 10 в [23]): $2245,2223.3$ и $2205.3 \mathrm{~cm}^{-1}$, что хорошо согласуется с расчетом $\left({ }^{12} \mathrm{C},{ }^{35} \mathrm{Cl}\right): 2246.3,2221.3$ и $2202.9 \mathrm{~cm}^{-1}$. Рисунок 4 иллюстрирует важную область, где наблюдается значительная вариабельность спектров в зависимости от изотопозамещения. Эта область спектра изучалась в работе [23] (спектрограмма 3), где также наблюдается высокий уровень корреляции с нашим теоретическим расчетом.

Наш опыт показывает [68], что трехквантовые переходы зачастую имеют невозмущенную интенсивность, достаточную для экспериментального наблюдения. Действительно, для основной молекулы, например, обнаруженное экспериментально при анализе спектра высокого разрешения состояние $v_{4}+2 v_{9}\left(1143.6684 \mathrm{~cm}^{-1}\right)$ [39] имеет предсказанную интенсивность $0.05 \mathrm{~km} / \mathrm{mol}$ и частоту $1136.7 \mathrm{~cm}^{-1}$ при отсутствии заимствования интенсивности от ближайших „светлых“ полос. Близкий по частоте переход $3 v_{9}\left(1098.7691 \mathrm{~cm}^{-1}\right)$ [39] также имеет малую, но достаточную для наблюдения собственную теоретическую интенсивность $0.004 \mathrm{~km} / \mathrm{mol}$ $\left(1093.0 \mathrm{~cm}^{-1}\right)$ и также не имеет предсказанного заимствования интенсивности.

\section{Заключение}

Фреон-22 является традиционным объектом пристального внимания со стороны экспериментальной колебательной и колебательно-вращательной спектроскопии в ИК диапазоне. При этом для ${ }^{13} \mathrm{C}$-замещенных изотопологов $\left({ }^{35} \mathrm{Cl},{ }^{37} \mathrm{Cl}\right)$ отсутствуют опубликованные исследования структур колебательно-вращательных полос высокого разрешения, а последняя работа по ангармоническому колебательному анализу датирована 1995 г. [24].

Данное исследование является первой публикацией в серии работ, посвященных резонансным полиадам в широком диапазоне колебательных переходов и анализу колебательно-вращательных полос ${ }^{13} \mathrm{C}$ замещенных изотопологов фреона-22 в области частот 1400$740 \mathrm{~cm}^{-1}$. С помощью гибридного метода было рассчитано неэмпирическое ангармоническое секстичное силовое поле, обеспечивающее совпадение предсказанных фундаментальных частот для двух ${ }^{12} \mathrm{C}$ изотопологов с погрешностью порядка $1.5 \mathrm{~cm}^{-1}$. Это позволило сделать прогноз изотопических сдвигов для изотопологов ${ }^{13}$ C. Расчет позволил выявить наиболее вариабельные области спектра, в которых изотопозамещение дает максимальное разделение пиков (например, 1650-1550 и $\left.850-750 \mathrm{~cm}^{-1}\right)$.

Ангармонический анализ четырех молекул также предоставил подробную информацию о характере и силе резонансных эффектов, что, в свою очередь, необходимо для интерпретации многоквантовых переходов. В частности, неэмпирический расчет уверенно прогнозирует и объясняет сильный случайный резонанс $v_{1} / v_{2}+v_{7}+v_{9}$ в основной молекуле, приводящий к образованию дублета 3024.55 и $3021.27 \mathrm{~cm}^{-1}$. Продемонстрирована эффективность использованной методики получения гибридного силового поля и на основе его решения прямой ангармонической колебательной задачи.

Проведенный анализ позволил предварительно идентифицировать центры колебательно-вращательных полос ${ }^{13} \mathrm{CHF}_{2}{ }^{35} \mathrm{Cl}$ и ${ }^{13} \mathrm{CHF}_{2}{ }^{37} \mathrm{Cl}$ в спектре смеси в рамках подготовки к индивидуальным анализам колебательновращательных структур отдельных колебательных переходов.

\section{Благодарности}

Авторы благодарны М.Н. Поповой за предоставленную возможность провести спектроскопические измерения высокого разрешения на оборудовании Лаборатории фурье-спектроскопии ИСАН и поздравляют ее с юбилеем.

\section{Конфликт интересов}

Авторы заявляют, что у них нет конфликта интересов.

\section{Список литературы}

[1] N. Ishikawa, Y. Kobayashi. Fluorine compounds - chemistry and application (Kodansha Scientific, Tokyo, 1979); [Н. Иcикава, Ё. Кобаяси. Фтор. Химия и применение (Мир, Москва, 1982)]

[2] A. Goldman, F.J. Murcray, R.D. Blatherwick, F.S. Bonomo, F.H. Murcray, D.G. Murcray. Geophys. Res. Lett., 8 (9), 1012 (1981). DOI: 10.1029/GL008i009p01012

[3] M.A.K. Khalil \& R.A. Rasmussen. Nature, 292, 823 (1981). DOI: $10.1038 / 292823 \mathrm{a} 0$

[4] C.P. Rinsland, D.W. Johnson, A. Goldman, J.S. Levine. Nature, 337, 535 (1989). DOI:10.1038/337535a0

[5] Global Ozone Research and Monitoring Project № 16, „Atmospheric Ozone“. World Meteorological Organization, Geneva. 1985.

[6] R. Zander, E. Mahieu, Ph. Demoulin, C.P. Rinsland, D.K. Weisenstein, M.K.W. Ko, N.D. Sze, M.R. Gunson. J. Atmos. Chem., 18 (2), 129 (1994). DOI: $10.1007 / \mathrm{BF} 00696811$

[7] C.P. Rinsland, A. Goldman, F.J. Murcray, R.D. Blatherwick, J.J. Kosters, D.G. Murcray, N.D. Sze, S.T. Massie. J. Geophys. Res., 95 (D10), 16477 (1990). DOI: $10.1029 / J D 095 i D 10 p 16477$

[8] P.C. Kruger, P. Fabian. Ber. Bunsenges. Phys. Chem., 90 (11), 1062 (1986). DOI: 10.1002/bbpc.19860901126 
[9] D.T. Cramb, Y. Bos, H.M. Jemson, M.C.L. Gerry, C.J. Marsden. J. Mol. Struct., 190, 387 (1988). DOI: $10.1016 / 0022-2860(88) 80298-9$

[10] Z. Kisiel, L. Pszczolkowski, G. Cazzoli, G. Cotti. J. Mol. Spectrosc., 173 (2), 477 (1995). DOI: $10.1006 /$ jmsp.1995.1250

[11] S. Blanco, A. Lesarri, J.C. López, J.L. Alonso, A. Guarnieri. Z. für Naturforsch. A, 51 (1-2), 129 (1996). DOI: $10.1515 /$ zna-1996-1-219

[12] N. Vogt, J. Demaison, H.D. Rudolph. Mol. Phys., 112 (22), 2873 (2014). DOI: 10.1080/00268976.2014.915067

[13] E.K. Plyler, W.S. Benedict. J. Res. Nat. Bur. Stand., 47 (3), 202 (1951). DOI: 10.6028/jres.047.026

[14] J.G. McLaughlin, M. Poliakoff, J.J. Turner. J. Mol. Struct., 82 (1-2), 51 (1982). DOI: 10.1016/0022-2860(82)85233-2

[15] F. Ito. Chem. Phys., 369 (2-3), 82 (2010). DOI: $10.1016 /$ j.chemphys.2010.02.020

[16] W. Holzer, H. Moser. J. Mol. Spectrosc., 20 (2), 188 (1966). DOI: 10.1016/0022-2852(66)90053-1

[17] A. Melchior, I. Bar, S. Rosenwaks. J. Phys. Chem. A, 102 (37), 7273 (1998). DOI: $10.1021 /$ jp982311q

[18] G. Glockler, J.H. Bachmann. Phys. Rev., 55 (7), 669 (1939). DOI:10.1103/PhysRev.55.669

[19] G. Glockler, G.R. Leader. J. Chem. Phys., 8 (9), 699 (1940). DOI: $10.1063 / 1.1750740$

[20] J.H. Lefebvre, A. Anderson. J. Raman Spectrosc., 23 (5) 243 (1992). DOI: 10.1002/jrs.1250230502

[21] Y.H. Wu, M. Onomichi, S. Sasaki, H. Shimizu. J. Raman Spectrosc., 24 (12), 845 (1993). DOI: $10.1002 /$ jrs. 1250241205

[22] A. Anderson, A.J. Beardsall, J.M. Fraser. Phys. Stat. Sol. (b), 182 (1), 59 (1994). DOI: 10.1002/pssb.2221820106

[23] A. Brown, D.C. McKean, J.L. Duncan. Spectrochim. Acta A., 44 (6), 553 (1988). DOI: 10.1016/0584-8539(88)80108-9

[24] P. Palmieri, R. Tarroni, M.M. Hühn, N.C. Handy, A. Willetts. Chem. Phys., 190 (2-3), 327 (1995). DOI: $10.1016 / 0301-0104(94) 00316-3$

[25] G.M. Kuramshina, F. Weinhold, I.V. Kochikov, A.G. Yagola, Yu.A. Pentin. J. Chem. Phys., 100 (2), 1414 (1994). DOI: $10.1063 / 1.466619$

[26] J.V. Magill, K.M. Gough, W.F. Murphy. Spectrochim. Acta A., 42 (6), 705 (1986). DOI:10.1016/0584-8539(86)80088-5

[27] A. Amrein, D. Luckhaus, F. Merkt, M. Quack. Chem. Phys. Lett., 152 (4-5), 275 (1988). DOI: $10.1016 / 0009-2614(88) 80092-7$

[28] A.J. Ross, A. Amrein, D. Luckhaus, M. Quack. Mol. Phys., 66 (6), 1273 (1989). DOI:10.1080/00268978900100871

[29] D. Luckhaus, M. Quack. Mol. Phys., 68 (3), 745 (1989). DOI: $10.1080 / 00268978900102511$

[30] A. Gambi, P. Stoppa, S. Giorgianni, A. de Lorenzi, R. Visinoni, S. Ghersetti. J. Mol. Spectrosc., 145 (1), 29 (1991). DOI:10.1016/0022-2852(91)90348-E

[31] G. Klatt, G. Graner, S. Klee, G. Mellau, Z. Kisiel, L. Pszczółkowski, J.L. Alonso, J.C. López. J. Mol. Spectrosc., 178 (1), 108 (1996). DOI: 10.1006/jmsp.1996.0163

[32] G.T. Fraser, J. Domenech, M.-L. Junttila, A.S. Pine. J. Mol. Spectrosc., 152 (2), 307 (1992). DOI: 10.1016/0022-2852(92)90071-U

[33] I. Merke, G. Graner, S. Klee, G. Mellau, O. Polanz. J. Mol. Spectrosc., 173 (2), 463 (1995).

DOI: $10.1006 /$ jmsp.1995.1249
[34] Z. Kisiel, J.L. Alonso, S. Blanco, G. Cazzoli, J.M. Colmont, G. Cotti, G. Graner, J.C. López, I. Merke, L. Pszczółkowski, J. Mol. Spectrosc., 184 (1), 150 (1997). DOI: $10.1006 /$ jmsp.1997.7304

[35] M. Snels, G. D’Amico. J. Mol. Spectrosc., 209 (1), 1 (2001). DOI: $10.1006 /$ jmsp.2001.8403

[36] C.D. Thompson, E.G. Robertson, D. McNaughton. Chem. Phys., 279 (2-3), 239 (2002). DOI: $10.1016 / \mathrm{S} 0301-0104(02) 00454-8$

[37] C.D. Thompson, E.G. Robertson, D. McNaughton. Phys. Chem. Chem. Phys., 5 (10), 1996 (2003). DOI: 10.1039/B300689A

[38] S. Albert, H. Hollenstein, M. Quack, M. Willeke. Mol. Phys., 102 (14-15), 1671 (2004). DOI: $10.1080 / 00268970412331290643$

[39] C.D. Thompson, E.G. Robertson, D. McNaughton. Mol. Phys., 102 (14-15), 1687 (2004). DOI: $10.1080 / 00268970410001725785$

[40] S. Albert, H. Hollenstein, M. Quack, M. Willeke. Mol. Phys., 104 (16-17), 2719 (2006). DOI:10.1080/00268970600828991

[41] S. Albert, A.K. Keppler, H. Hollenstein, T.C. Manca, M. Quack. Handbook of High-Resolution Spectroscopy, ed. by M. Quack, F. Merkt (Wiley, Chichester, 2011) V. 1. Ch. 3. P. 117-173. DOI: 10.1002/9780470749593

[42] M. Gauthier, G.G. Cureton, P.A. Hackett, C. Willis. Appl. Phys. B., 28 (1), 43 (1982). DOI: 10.1007/BF00693891

[43] A. Outhouse, P. Lawrence, M. Gauthier, P.A. Hackett. Appl. Phys. B., 36 (2), 63 (1985). DOI: 10.1007/BF00694692

[44] P. Ma, K. Sugita, S. Arai. Appl. Phys. B., 50 (5), 385 (1990). DOI: $10.1007 / \mathrm{BF} 00325091$

[45] В.Н. Лохман, Г.Н. Макаров, Е.А. Рябов, М.В. Сотников. Квант. электрон., 23 (1), 81 (1996).

[46] W. Fuß, J. Göthel, M. Ivanenko, K.L. Kompa, W.E. Schmid. Z. für Phys. D., 24, 47 (1992). DOI:10.1007/BF01436603

[47] V.Yu. Baranov, A.P. Dyad'kin, D.D. Malyuta, V.A. Kuzmenko, S.V. Pigilsky, V.S. Mezhevov, V.S. Letokhov, V.B. Laptev, E.A. Ryabov, I.V. Yarovoy, V.B. Zarin, A.S. Podoryashy. Proc. of SPIE., 4165, 314 (2000). DOI: 10.1117/12.394137

[48] Изотопы: свойства, получение, применение, Т. 1. Под ред. Баранова В.Ю. (Физматлит, Москва, 2005), Разд. 8.4. C. 460 .

[49] R.I. Martinez, J.T. Herron. Chem. Phys. Lett., 84 (1), 180 (1981). DOI: 10.1016/0009-2614(81)85396-1

[50] A. Melchior, P. Knupfer, I. Bar, S. Rosenwaks, T. Laurent, H.-R. Volpp, J. Wolfrum. J. Phys. Chem., 100 (32), 13375 (1996). DOI: 10.1021/jp9609038

[51] L. Li, G. Dorfman, A. Melchior, S. Rosenwaks, I. Bar. J. Chem. Phys., 116 (5), 1869 (2002). DOI: 10.1063/1.1427915

[52] V.M. Apatin, V.O. Kompanets, V.B. Laptev, Yu.A. Matveets, E.A. Ryabov, S.V. Chekalin, V.S. Letokhov. Chem. Phys. Lett., 414 (1-3), 76 (2005). DOI: 10.1016/j.cplett.2005.07.116

[53] A. Amrein, H.-R. Dübal, M. Quack. Mol. Phys., 56 (3), 727 (1985). DOI:10.1080/00268978500102671

[54] G. Guelachvili, R.K. Narahari. Handbook of IR standards (Academic Press, Orlando, 1986).

[55] M.J. Frisch, G.W. Trucks, H.B. Schlegel, G.E. Scuseria, M.A. Robb, J.R. Cheeseman, G. Scalmani, V. Barone, B. Mennucci, G.A. Petersson et al., Gaussian 09, Revision B.01 (Gaussian, Inc., Wallingford CT, 2010). URL: http://www.gaussian.com/ 
[56] D. Papoušek, M.R. Aliev. Molecular Vibrational/Rotational Spectra (Academia, Prague, 1982).

[57] Ю.С. Макушкин, В.Г. Тютерев. Методы возмущений $u$ эфбективные гамильтонианы в молекулярной спектроскопии (Наука, Новосибирск, 1984).

[58] M.R. Aliev, J.K.G. Watson. Molecular Spectroscopy: Modern Research, ed. by Rao K.N. (Academic Press, New York, 1985). V. III. Ch. 1. P. 1-67.

[59] S.V. Krasnoshchekov, E.V. Isayeva, N.F. Stepanov. J. Phys. Chem. A., 116 (14), 3691 (2012). DOI: 10.1021/jp211400w

[60] S.V. Krasnoshchekov, N.F. Stepanov. J. Chem. Phys., 139 (18), 184101 (2013). DOI: 10.1063/1.4829143

[61] S.V. Krasnoshchekov, E.V. Isayeva, N.F. Stepanov. J. Chem. Phys., 141 (23), 234114 (2014). DOI: 10.1063/1.4903927

[62] S.V. Krasnoshchekov, N.C. Craig, N.F. Stepanov. J. Phys. Chem. A, 117 (14), 3041 (2013). DOI: 10.1021/jp311398z

[63] S.V. Krasnoshchekov, N. Vogt, N.F. Stepanov. J. Phys. Chem. A, 119 (25), 6723 (2015).

DOI: $10.1021 /$ acs.jpca.5b03241

[64] S.V. Krasnoshchekov, N.C. Craig, P. Boopalachandran, J. Laane, N.F. Stepanov. J. Phys. Chem. A, 119 (43), 10706 (2015). DOI: 10.1021/acs.jpca.5b07650

[65] S.V. Krasnoshchekov, R.S. Schutski, N.C. Craig, M. Sibaev, D.L. Crittenden. J. Chem. Phys., 148 (8), 084102 (2018). DOI: $10.1063 / 1.5020295$

[66] S.V. Krasnoshchekov, V.B. Laptev, I.K. Gainullin. J. Quant. Spectrosc. Radiat. Transfer, 217, 243 (2018).

DOI: $10.1016 /$ j.jqsrt.2018.06.004

[67] S.V. Krasnoshchekov, N.C. Craig, L.A. Koroleva, N.F. Stepanov. Spectrochim. Acta A, 189, 66 (2018).

DOI: $10.1016 /$ j.saa.2017.07.062

[68] C.A. Pietropolli, L. Bizzocchi, B.M. Giuliano, P. Caselli, N.C. Craig, S.V. Krasnoshchekov. JQSRT, 239, 106656 (2019). DOI: $10.1016 /$ j.jqsrt.2019.106656

[69] S.V. Krasnoshchekov, V.B. Laptev, S.A. Klimin, I.K. Gainullin, A.A. Makarov. Spectrochim. Acta A, 238, 118396 (2020). DOI: 10.1016/j.saa.2020.118396

[70] O.L. Polyansky, R.I. Ovsyannikov, A.A. Kyuberis, L. Lodi, J. Tennyson, N.F. Zobov. J. Phys. Chem. A, 117 (39), 9633 (2013). DOI: 10.1021/jp312343z

[71] M.E. Kellman. J. Chem. Phys., 93 (9), 6630 (1990). DOI: $10.1063 / 1.458930$

[72] M.W. Mackenzie. Spectrochim. Acta A., 40 (3), 279 (1984). DOI: $10.1016 / 0584-8539(84) 80049-5$

[73] S.V. Krasnoshchekov, E.O. Dobrolyubov, M.A. Syzgantseva, R.V. Palvelev. Mol. Phys., 118 (11), e1743887 (2020).

DOI: $10.1080 / 00268976.2020 .1743887$ 